laterale, non hypertrophié en volume, l'aspect de la trame conjonctive paraît être équivalent pour les deux races, où elle présente, entre animaux, une variation du même ordre de grandeur. La taille des myoskhènes est nettement plus développée, chez le Piétrain, dans le cas des muscles qui manifestent une hypertrophie. Mais elle est pratiquement identique pour les muscles non hypertrophiés où l'on retrouve chez le Piétrain, une structure musculaire similaire à celle des Large-White. D'autre part, l'hypertrophie en volume s'accompagne d'une augmentation notable du diamètre des fibres.

Ces observations confirment, sur le plan histologique, l'hypothèse selon laquelle la race Piétrain présenterait une hypertrophie musculaire de même importance sinon plus accentuée que celle, observée chez les bovins, dans le cas des animaux dits "culard " ou " cul-de-poulain ". On peut en effet avancer que, dans les zones où se manifestent une hypertrophie en volume d'un muscle, sa structure se modifie de telle manière que, simultanément, l'importance relative de la trame de tissu conjonctif (par rapport à l'ensemble des fibres musculaires) diminue et que les dimensions des éléments principaux de son réseau - qui constituent les myoskhènes augmentent. L'ampleur de la manifestation conjointe de ces deux phénomènes pourrait être retenue pour évaluer l'hypertrophie affectant un muscle à un niveau déterminé.

\title{
DÉVELOPPEMENT MUSCULAIRE E'T TAUX D'HYDROXYPROLINE DU MUSCLE DANS LES RACES PORCINES LARGE-WHITE ET PIETRAIN
}

\author{
P. SELIIER. — Station de Génétique quantitative et appliquée, C.N.R.Z., 78-Jouy-en-Josas. \\ R. BOCCARD. - Station de Recherches sur la Viande, C.R.Z.V.R., \\ 63-Theix par St-Genès-Champanelle.
}

L'hypertrophie musculaire, dans les espèces bovine et porcine, s'accompagne d'une réduction du taux d'hydroxyproline du muscle, qui traduit une moindre teneur en tissu conjonctif. L'objet de cette étude est d'étudier la contribution que peut apporter la mesure du taux d'hydroxyproline à la recherche de critères analytiques permettant de définir objectivement l'hypertrophie musculaire du porc de Piétrain par rapport à un type normal représenté ici par la race Large-White. 77 porcs de Pietrain et 55 porcs de la race Large-White ont été abattus à un poids voisin de $95 \mathrm{~kg}$. Sur chaque porc, le taux d'hydroxyproline $\left(\mathrm{X}_{1}\right)$ du muscle Triceps brachii caput laterale (anconé externe) a été déterminé : il est exprimé par le rapport de l'azote de l'hydroxyproline à l'azote total du tissu musculaire. D'autre part, 12 mesures de carcasse ont été considérées. La distribution de fréquence de $\mathrm{X}_{1}$ s'écarte significativement de la normalité dans l'échantillon de la race de Piétrain; son allure bimodale suggère l'existence, à l'intérieur de cette population, de deux types d'animaux pour la teneur du muscle en collagène. La normalité de la loi de la variable $\mathrm{X}_{1}$ en race de Piétrain a cependant été admise dans la suite de l'étude.Dans les 2 races, le taux d'hydroxyproline est inférieur chez les animaux mâles $(\mathrm{P}<0,05)$. L'effet "race " est hautement significatif $(P<0,01): 3,93 \pm 0,05$ p. 1000 en race de Piétrain contre 4,35 \pm 0,06 p. 1000 en race Large-White. Les corrélations entre $\mathrm{X}_{1}$ et les autres caractères de carcasse sont toutes faibles et non significatives à l'exception d'une corrélation négative entre $\mathrm{X}_{1}$ et le dépôt de lard dorsal en race de Piétrain. Une fonction linéaire a été établie à partir de 6 variables choisies parmi les 13 variables considérées de manière à permettre la meilleure discrimination entre les 2 races. La fonction obtenue est de la forme suivante : Y \# longueur cm - 4 (jambon + longe + poitrine) $\mathrm{kg}+1 / 4$ (lard cou - lard dos) mm. Cette fonction assure une discrimination presque totale entre le type de Piétrain et le type normal Large-White mais les coefficients de corrélation entre $\mathrm{X}_{1}$ et $\mathrm{Y}(0,10$ en race de Piétrain et 0,20 en race Large-White) ne sont pas significatifs. L’ensemble de ces résultats indique que, dans les deux races considérées, le développement musculaire et la réduction du collagène dans le muscle apparaissent comme deux phénomènes pratiquement indépendants. 